\title{
Methodological Approaches for Dietary Intake Assessment in Formula-fed Infants
}

\author{
*Veronica Luque, *Joaquin Escribano, *Georgina Mendez-Riera, ${ }^{\dagger}$ Sonia Schiess, \\ ${ }^{\dagger}$ Berthold Koletzko, ${ }^{\ddagger}$ Elvira Verduci, ${ }^{\S}$ Anna Stolarczyk, ${ }^{\prime}$ Françoise Martin, and \\ * Ricardo Closa-Monasterolo
}

\begin{abstract}
Background and Objective: The estimation of dietary intake remains a challenge in human nutritional studies. In infants, the use of food diaries (FDs) is a suitable method already validated; however, this method is not exempt from error. In formula-fed infants, dilution of powdered formulas may produce errors. Our aim was to develop and validate standard operating procedures (SOPs) for the assessment of dietary intake in formula-fed infants, attending potential sources of error.

Methods: We analysed sources of error in a random subsample of 3-day FDs from 100 infants enrolled in the European Childhood Obesity Project. Calculations to estimate intakes were standardised and included in a software tool (SOPsystem). An evaluation of a simulated FD was performed by 9 trained nutritionists and 23 nutrition students $(n=225$ and $\mathrm{n}=575$ bottle evaluations, respectively) to compare the results obtained when using or not the SOPsystem. Correct answers (\%), coefficients of
\end{abstract}

Received July 5, 2012; accepted September 23, 2012.

From the *Universitat Rovira i Virgili, IISPV, Reus, Spain, the †Hauner Children's Hospital, University of Munich Medical Centre, Munich, Germany, the ‡Department of Pediatrics, University of Milan, San Paolo Hospital, Milan, Italy, the §Children's Memorial Health Institute, Warsaw, Poland, and the $\| \mathrm{CHC}$ St Vincent Liège, Belgium.

Address correspondence and reprint requests to Ricardo Closa-Monasterolo, Paediatrics Unit, Universitat Rovira i Virgili, IISPV, C/Sant Llorenç 21, 43201 Reus, Spain (e-mail: ricardo.closa@urv.cat). www.clinicaltrials.gov registration number: NCT00338689.

European Childhood Obesity Project Study Group members: Grote V, Koletzko B, Beyer J, Demmelmair H, Fritsch M, Haile G, Handel U, Hannibal I, Pawellek I, Schiess S, Verwied-Jorky S, von Kries R, Weber M (Children's University Hospital, University of Munich Medical Centre, Munich, Germany), Escribano J, Closa R, Luque V, Ferre N, Zaragoza-Jordana M, Mendez-Riera G (Pediatrics Unit, Universitat Rovira i Virgili, IISPV, Reus, Spain), Gruszfeld D, Socha P, Socha J, Dobrzańska A, Janas R, Wierzbicka A (Children's Memorial Health Institute, Warsaw, Poland), Carlier C, Dain E, Goyens P, Hoyos J, Poncelet P (ULB, Bruxelles, Belgium), Xhonneux A, Van Hees JN, Langhendries J-P (CHC St Vincent Liege, Belgium), Perrin E (Danone Research Centre for Specialized Nutrition, Schiphol, The Netherlands), Agostoni C, Giovannini M, Re Dionigi A, Riva E, Scaglioni S, Vecchi F, Verducci E (University of Milan).

The European Childhood Obesity Project was carried out with the financial support of the European Community, under the 5th Framework Programme for Research, Technology \& Demonstration "Quality of Life and Management of Living Resources," Key Action 1 (Food, Nutrition \& Health), contract number QLK1-CT2002-389. B.K. is the recipient of a Freedom to Discover Award from the Bristol-Myers Squibb Foundation, New York, NY. This article does not necessarily reflect the views of the Commission and in no way anticipates its future policy in this area.

The authors report no conflicts of interest.

Copyright $(1) 2013$ by European Society for Pediatric Gastroenterology, Hepatology, and Nutrition and North American Society for Pediatric Gastroenterology, Hepatology, and Nutrition

DOI: 10.1097/MPG.0b013e3182779a60 variation, and the time spent (minutes) to assess the simulated FD were analysed.

Results: Overall, $60 \%$ of the FDs contained reports of incorrect volumes, and $37 \%$ reported incorrect formula dilutions. When the SOPsystem was used, correct answers in the simulated FD were more frequent $(P<0.001)$ and the mean coefficient of variation and the time spent were lower $(P<0.005$ and $P<0.01$, respectively).

Conclusions: The development and implementation of SOPs with a software tool that identifies specific sources of error in record-keeping achieved a harmonised and improved process for assessing dietary intakes in formula-fed infants, minimising errors in calculations and reducing the work time invested.

Key Words: dietary intake assessment, infant feeding, infant formula, multicentre trials, validation study

(JPGN 2013;56: 320-327)

$\mathrm{T}$ he estimation of dietary intake remains a challenge in human nutritional studies. Methods available to collect information about dietary intake are imprecise $(1,2)$. Nutritionists need to develop specific systems and standard operating procedures (SOPs) to ensure the maximum accuracy and quality of data, taking into account factors such as the target population and the aim of the evaluation (3). Typical sources of error when researchers and clinicians try to assess dietary intake are participants' misreporting, incomplete information in food diaries (FDs), errors in interpretation and calculations transferring the reported data to the database, and difficulties in finding appropriate nutrient composition databases (1). In children, additional sources of error can influence the accuracy of dietary intake assessments. These sources of error depend particularly on the child's age (including their ability to estimate portions, their capacity to recall food intake, the use parents as proxy reporters) $(1,4)$. Training participants in reporting dietary intake as well as training nutritionists in revising the reported information (extracting as many details as possible) are essential for obtaining accurate data.

These rules were taken into account within the European Childhood Obesity Project, a large multicentre randomised clinical trial to examine the effects of protein intake during the first months of life on later obesity risk (5). In 5 countries (Germany, Belgium, Italy, Poland, and Spain), 1138 formulafed healthy infants were recruited and randomised into 2 arms receiving powdered study formulas with lower or higher protein content, according to the European Commission Directive (6). The dietary intake of participating infants was recorded by parents, who completed ten 3-day FDs during the first year of the child's life (7). This technique has been identified as the 
method that provides the best estimates in this age range (8), where parents and caregivers are responsible for recording the infant's dietary intake $(2,9)$.

As for all dietary assessment methods, FDs are not exempt from error and bias from the criterion standard methods (10). In such a longitudinal multicentre study, there was a need to develop and implement SOPs with regard to data collection, the checking and interpretation of FDs, and the calculation and data introduction into the food database. Although there are validated methods for assessing the dietary intake of adults, there is a need for validated methods for assessing dietary intake in infancy and childhood $(2,4)$. Specifically, in the present study, potential sources of error in measuring the dietary intake of infants who are fed reconstituted powdered formulas have been identified. Formula dilution, the interpretation of FDs by nutritionists, and the sequence of calculations needed to quantify the consumed amount of formula may all contribute to errors in the dietary assessment of formula-fed infants. For instance, parents do not always dilute powdered formulas according to the manufacturers' instructions, which may lead to a reconstituted formula with an unknown nutrient density. Indeed, a systematic review identified errors in the reconstitution of a powdered formula milk preparation in all identified studies conducted in the United Kingdom (11). The present practices for the preparation of infant formula in other European countries (where the study was being conducted) are not known.

Another particular source of potential errors is that parents may not consider the increase in volume caused by the addition of powdered formula to water, which results in a larger volume of prepared formula than the amount of water used. Therefore, we considered it necessary to design specific FDs to allow proper data collection and checking and to standardise the procedures for FD interpretations and calculations in our multicentre study to reduce potential sources of error. Here we report on the frequency of errors made in diluting powdered formula and in reporting the total volume of formula milk by parents and caregivers, as well as the approach used to detect these errors and the experience gathered.

The aim of the present study was to develop, validate, and ensure the proper use of SOPs to assess the dietary intake of formula-fed infants, while identifying and correcting for potential sources of error.

\section{METHODS}

A parallel cross-sectional observational study to validate the methods used to assess dietary intakes in formula-fed infants was designed.

\section{Collection of Dietary Intake Data}

A 3-day weighed FD was used to collect formula intake during the first year of life in the 5 countries taking part in the Childhood Obesity Project. Parents were asked to record everything consumed by the infant during a 3-day period ( 2 weekdays and 1 weekend day). FDs were designed to track bottle preparation (amounts of water, powdered formula and powdered cereals, where applicable) and the total offered and actually consumed volumes. This specially designed FD, where parents were asked to record all the details of the preparation of each bottle, allowed the nutritionists to detect cases in which parents diluted the formula deviating from manufacturers' instructions, as well as cases in which water volume was reported instead of total offered volume. Further details have been published elsewhere $(7,12)$.

\section{Identification of Specific Sources of Error in the Preparation and Recording of Intakes of Infant Formula Milk by Parents and Caregivers}

A random subsample of FDs that were kept between October 2002 and July 2005, during the first year of life of 100 infants participating in the study (20 infants from each country, ten 3-day FDs from each infant), was blindly selected (investigators from each study centre randomly took 20 children's folders without seeing any identifier). Using the diaries, the frequency of parental errors in diluting powdered formula milk and in reporting total milk volumes was analysed.

Nutritionists from the 5 study centres checked every single diary following the SOPs document to identify

1. Whether at least 1 bottle was prepared by parents or caregivers who did not follow the manufacturers' instructions (leading to an infant formula of unknown nutritional composition).

2. Whether at least the total volume of one bottle was recorded without taking into account the volume increase produced by the addition of powdered formula and cereals.

The specific source of errors in reporting infant dietary intakes by parents or caregivers is analysed as a percentage of FDs with incorrectly prepared bottles (by the incorrect dilution of powdered formula) and the percentage of FDs with no correct total volumes offered and consumed.

\section{Nutritionists' SOPs}

The SOPs were developed to ensure a harmonised methodological approach by the nutritionists at all centres while evaluating the dietary protocols. To determine the mean volume increase by the addition of powdered formula and cereals to water, 10 tests were performed. In these tests, a mean weight of $4.7 \mathrm{~g}$ of formula was added to $30 \mathrm{~mL}$ of water in test tubes with a precision of $1 \mathrm{~mL}$. The process was repeated for 6 different infant formulas and 3 different kinds of cereals. The established standard increase in volumes was $3 \mathrm{~mL}$ for each addition of one level scoopful of powdered formula and $6.5 \mathrm{~mL}$ for the addition of $10 \mathrm{~g}$ of powdered cereals.

Equations and procedures were established for different situations that could induce error, such as combinations of correct or incorrect formula reconstitution with incorrect recording of total volume. The following example illustrates the procedure:

If the preparation was $240 \mathrm{~mL}$ of water with 7 scoopfuls of powdered formula, the total amount prepared was $260 \mathrm{~mL}$ and the remaining volume was $65 \mathrm{~mL}$. Thus, the total amount consumed would be $195 \mathrm{~mL}$; however, the $195 \mathrm{~mL}$ of formula milk would have a nutritional composition unknown. Therefore, it is needed to translate $195 \mathrm{~mL}$ consumed into the corresponding volume of formula in which the nutrient composition is known. The needed calculations for this translation would be

1. The actual nutrient value of 7 formula scoopfuls would correspond to a preparation with $210 \mathrm{~mL}$ of water (to give a total volume of approximately $231 \mathrm{~mL}$ )

2. The infant consumed $75 \%$ of the bottle $(195 \mathrm{~mL}$ of $260 \mathrm{~mL})$

3. Seventy-five percent of $231 \mathrm{~mL}$ is $173.25 \mathrm{~mL}$.

Therefore, $195 \mathrm{~mL}$ of an unknown nutrient composition formula would be translated to approximately $173 \mathrm{~mL}$ of formula milk of known nutrient composition.

Or more briefly, in only 1 step: 
Formula intake $\left(\mathrm{Vol}_{\mathrm{FI}}\right)$ would be equal to [Vol $\mathrm{VTD}_{\mathrm{STO}} \times$ $\left.\mathrm{Vol}_{\mathrm{CO}}\right] / \mathrm{Vol}_{\mathrm{OF}}$,

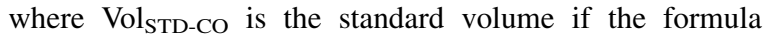
is correctly diluted $(231 \mathrm{~mL}), \mathrm{Vol}_{\mathrm{CO}}$ is the consumed volume $(195 \mathrm{~mL})$, and $\mathrm{Vol}_{\mathrm{OF}}$ is the offered volume $(260 \mathrm{~mL})$.

Table 1 shows the main equations included in the SOPs protocol used to calculate dietary intakes from food records containing formula milk not properly reported or diluted.

Because several variables are needed to complete the calculations (millilitres of water, number of scoopfuls, grams of cereals, offered volume, remaining volume), it is extremely important to perform a proper check of the FD with the reporters to obtain the highest data quality. Mandatory variables required to properly calculate nutrient intake are water volume, number of scoopfuls, and remaining volume. When checking the diary with the reporters is not possible, the assumptions to be made were standardised in the SOPs protocol. In the present study, when incorrect dilution information (the number of scoopfuls did not correspond to the water volume) was reported in the FDs, but it was not possible to check with the study participants, it was assumed to be a dilution error, rather than a typing error. In the case of inconsistency between the amount of ingredients and the offered volume, the protocol gave priority to the variables of water volume and number of scoopfuls rather than to the offered volume (because this was considered a frequent source of error, when the reporters did not take into account the increase in volume by the addition of powder to water).

To reduce potential errors in the nutritionists' calculations as well as the time spent on the dietary assessment process, we developed a tool using Excel for Windows XP software (Microsoft; SOPsystem; Microsoft, Redmond, WA). The SOPsystem was developed considering the standard volumes and calculation procedures previously established in the protocol. Only the amounts of all bottle ingredients (water, powdered formula, and cereals) and the volume remaining after a meal needed to be entered in SOPsystem, and amounts of actual dietary intakes were then calculated by the software.

Nutritionists from the 5 different countries participating in the multicentre trial were trained to check and interpret the FDs, calculate dietary intakes following established standards, and use the SOPsystem.

\section{Validation of the SOPs and the SOPsystem}

To validate the SOPsystem, a simulated FD consisting of 25 bottles ( 9 of which also contained cereals) was designed.

\section{Implementation of the SOPsystem by Trained Nutritionists}

Nine trained nutritionists working on the project were invited to test the simulated FD interpretation and calculations using 2 different methods: performing the calculation using the SOPsystem or not using the SOPsystem for calculations, on the same 25 bottlesimulated diary. The results of this training were analysed and are described below.

\section{Implementation of the SOPsystem by Nonexpert Nutritionists}

As part of a nutrition university degree training session in dietary assessment methods in infants, a group of 23 Spanish nutrition university students not trained in infant formula intake assessment also performed the interpretation and calculation using the simulated FD. The students participated in an initial session with a brief explanation of the increase in volume produced by the addition of powdered milk and cereals to the water and different sources of error in infant formula intake assessment. At the end of this session, the nutrition students used a calculator to analyse the simulated FD. In a second session, they calculated the same simulated FD using the SOPsystem, after receiving an explanation about the software programme.

The validity of the implementation of the method was evaluated as the percentage of correct and incorrect answers. The correct volumes of formula for the simulated diary were agreed upon by 3 of the nutritionists trained in using the SOPs. Answers within $\pm 2 \%$ of the agreed value were considered to be correct. This range around the agreement value was defined to determine a minimum error produced by decimal positions in calculations, but to avoid any real error in calculations produced by investigators. The ability of the SOPsystem to standardise nutritionists' evaluations was tested using internutritionists' variance coefficients. Additionally, the time spent to assess the simulated FD was analysed.

\section{Statistical Analysis}

Descriptive results are presented as the mean (95\% confidence interval). Kruskal-Wallis tests were used to assess differences by country in the parental reports of total volumes and formula dilutions. Differences in the frequency of correct answers using or not using the SOPsystem were evaluated by a McNemar test, using a $\pm 2 \%$ value obtained as the correct answer. Absolute risk reduction (\% [95\% confidence interval]) was calculated to assess the reduction in the number of bottles calculated incorrectly using the SOPsystem. To analyse the internutritionist variance in the estimations of formula intake, the coefficient of variation (CV) for each bottle was calculated. To compare the internutritionist variation between the 2 methods, a paired samples Student $t$ test or a Wilcoxon test (when not normally distributed) was performed. Normality was determined by the Kolmogorov-Smirnov $Z$ test. The time spent on the evaluation of the simulated diary was measured, and a paired samples Student $t$ test was used to compare the 2 methods. Statistical significance was accepted at $P<0.05$. SPPS version 17.0 (SPSS Inc, Chicago, IL) was used to perform the statistical analysis. The European Childhood Obesity Project was approved by the ethical committees of all study centres.

\section{RESULTS}

\section{Identification of Specific Sources of Error in Parental and Caregiver Preparation and Recording of Infants' Formula Milk Intake}

The analysis of the dietary protocols from the random subgroup of 100 infants (20 from each of the 5 countries) showed that $60 \%$ of parents reported incorrect total amounts of formula offered (ie, they did not take into account increasing volumes) in at least 1 of the FDs. Figure 1 shows the frequency of incorrectly reported total volumes in the random subsample at each time point. In the entire sample, the mean proportion of bottles incorrectly reported was $50.6 \%(43.0-58.1)$. There were significant differences by country in the frequency of incorrect reporting by parents or caregivers $(P<0.001)$. Parents incorrectly reported the total volume for $19.6 \%$ of the bottles in Germany, $38.0 \%$ in Belgium, $74.4 \%$ in Italy, $60.6 \%$ in Poland, and $59.9 \%$ in Spain. This evaluation also indicated that $37 \%$ of parents did not follow the manufacturers' instructions for the dilution of infant formula in at least one of the FDs. Figure 2 shows the frequency of incorrectly 
TABLE 1. Equations included in the SOPs and SOPsystem to calculate dietary intakes from reported information in food diaries, attending potential source of error

Potentially misleading situations

to be detected in food diaries

Standard operating procedures and equations

Total volume reported without taking into account the increasing volume by adding powdered formula to the water

Bottle of formula milk incorrectly prepared (prepared not following the instructions of the manufacturers)

Cereals added to the formula milk (thus, part of the total $\mathrm{Vol}_{\mathrm{OF}}$ came from cereals and a part came from formula milk) a) If the infant consumed the full amount of it $\left(\mathrm{Vol}_{\mathrm{OF}}=\mathrm{Vol}_{\mathrm{FI}}\right)$

To find out the standard volume correspondent to the number of powdered formula scoopfuls used to prepare the bottle. If 1 scoopful of powdered formula $\approx 3 \mathrm{~mL}$,

$\mathrm{Vol}_{\mathrm{FI}}=\mathrm{Vol}_{\mathrm{STD}}=([\mathrm{n}$ scoopfuls $\times 3 \mathrm{~mL}]+[\mathrm{n}$ scoopfuls $\times 30 \mathrm{~mL}])$

b) If the infant did not consume the full amount of it

To find out the standard volume correspondent to the number of powdered formula scoopfuls and to subtract the reported remaining volume

$\mathrm{Vol}_{\mathrm{FI}}=\mathrm{Vol}_{\mathrm{STD}}-\mathrm{Vol}_{\mathrm{RE}}$

c) If the infant consumed the full amount of it

To find out the standard volume if the bottle would be correctly prepared

$\left(\mathrm{Vol}_{S T D-C P}\right)$ using the number of powdered formula scoopfuls added to prepare the bottle and the amount of water that should be added to that $\mathrm{n}$ scoopfuls

$\mathrm{Vol}_{\mathrm{FI}}=\mathrm{Vol}_{\mathrm{STD}-\mathrm{CP}}=([\mathrm{n} \mathrm{scoopfuls} \times 3 \mathrm{~mL}]+[\mathrm{n} \mathrm{scoopfuls} \times 30 \mathrm{~mL}])$

d) If the infant did not consume the full amount of it

1st step: to find out the volume of consumed formula (not correctly prepared)

$\mathrm{Vol}_{\mathrm{CO}}=(\mathrm{mL}$ of water added to bottle $+[\mathrm{n}$ scoopfuls $\times 3 \mathrm{~mL}])-\mathrm{Vol}_{\mathrm{RE}}$

2nd step: To find out the milk volume (of known nutrient composition) correspondent to $\mathrm{Vol}_{\mathrm{CO}}$

$\mathrm{Vol}_{\mathrm{FI}}=\left(\mathrm{Vol}_{\mathrm{STD}-\mathrm{CP}} \times \mathrm{Vol}_{\mathrm{CO}}\right) / \mathrm{Vol}_{\mathrm{OF}}$

e) If the formula was correctly prepared

1st step: to calculate the volume increased by adding cereals to the bottle

$10 \mathrm{~g}$ cereals $=6.5 \mathrm{~mL} \rightarrow \mathrm{Vol}_{\mathrm{CE}}=\mathrm{g}$ of cereals added $\times 0.65$

2nd step: to find out the total volume consumed

$\mathrm{Vol}_{\mathrm{BOT}}=\mathrm{Vol}_{\mathrm{OF}}+\mathrm{Vol}_{\mathrm{CE}}$

3rd step: to calculate the percentage consumed (from total prepared bottle)

$\left.\% \mathrm{Vol}_{\mathrm{BOT}}=\left[\left(\mathrm{Vol}_{\mathrm{BOT}}-\mathrm{Vol}_{\mathrm{RE}}\right) \times 100\right)\right] / \mathrm{Vol}_{\mathrm{BOT}}$

4th step: to apply the obtained percentage to the formula offered and the cereals separately

$\mathrm{Vol}_{\mathrm{FI}}=\left(\mathrm{Vol}_{\mathrm{OF}} \times \% \mathrm{Vol}_{\mathrm{BOT}}\right) / 100$

Cereals consumed $(\mathrm{g})=\left(\mathrm{g}\right.$ of cereals added to bottle $\left.\times \% \mathrm{Vol}_{\text {BOT }}\right) / 100$

f) If the formula was not prepared following manufacturers' instructions

1st step: to calculate $\mathrm{Vol}_{\mathrm{CE}}$

2nd step: to calculate $\mathrm{Vol}_{\mathrm{BOT}}$

3rd step: to calculate the percentage consumed (from total prepared bottle)

$\% \mathrm{Vol}_{\mathrm{BOT}}=\left(\left[\mathrm{Vol}_{\mathrm{BOT}}-\mathrm{Vol}_{\mathrm{RE}}\right] \times 100\right) / \mathrm{Vol}_{\mathrm{BOT}}$

4th step: to apply the obtained percentage to the formula offered if it would

be correctly prepared ( $\left.\mathrm{Vol}_{\mathrm{STD}}\right)$ and the cereals separately

$\mathrm{Vol}_{\mathrm{FI}}=\left(\mathrm{Vol}_{\mathrm{STD}-\mathrm{CP}} \times \% \mathrm{Vol}_{\mathrm{BOT}}\right) / 100$

Cereals consumed $(\mathrm{g})=\left(\mathrm{g}\right.$ of cereals added to the bottle $\left.\times \% \mathrm{Vol}_{\mathrm{BOT}}\right) / 100$

Vol $_{\mathrm{OF}}$ is the offered volume of formula milk (this is the total volume of the bottle offered, where formula can be diluted following manufacturers instructions or not), $\mathrm{Vol}_{\mathrm{FI}}$ is the final volume of formula milk consumed (which nutrient composition is known), Vol $\mathrm{STD}_{\mathrm{S}}$ is the standard volume of the bottle correspondent to the number of scoopfuls added to the bottle, $\mathrm{Vol}_{\mathrm{RE}}$ is the reported remaining volume, Vol $\mathrm{STD}_{\mathrm{CP}}$ is the standard volume of the bottle correspondent to the powdered formula if it would be correctly diluted ([n scoopfuls $\times 3]+[\mathrm{nmL}$ of water that should be added to $\mathrm{n}$ scoopfuls $\times 30]$ ), Vol $\mathrm{CO}_{\mathrm{B}}$ is the reported consumed volume of formula not correctly diluted (which nutrient composition is unknown), $\mathrm{Vol}_{\mathrm{BO}}$ is the total bottle volume, containing formula (correctly diluted or not) and powdered cereals diluted, $\mathrm{Vol}_{C E}$ is the volume increased by adding cereals to the bottle.

diluted formula milk in the same random subsample by time point. In the entire sample, $8.3 \%(5.1-11.4)$ of the bottles were incorrectly diluted. We did not find significant differences by country in formula dilution.

\section{Implementation of the SOPsystem}

The analyses of the efficacy of implementation of the SOPsystem among trained nutritionists and nonexperienced nutritionists showed higher rates of correct answers using the SOPsystem than without in all cases, mostly with statistically significant differences (Table 2). Table 2 shows the frequency among trained nutritionists of correct and incorrect estimates for formula and powdered cereals using the SOPsystem versus not using it. Table 3 shows the frequency of correct and incorrect estimates by the inexperienced nutrition students. Each test (n) represents 1 calculated bottle by 1 observer. In other words, the evaluation of 25 bottles each performed by 9 nutritionists represents 225 evaluations. Nine of the 25 bottles 


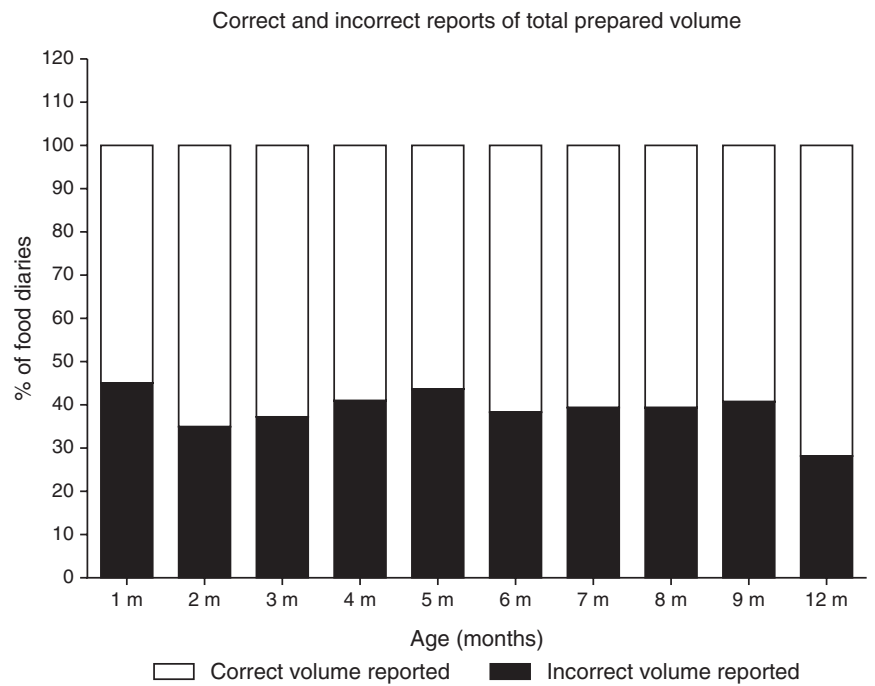

FIGURE 1. Frequency of correctly and incorrectly reported total volumes in a random subsample of 10 food diaries of 100 infants in the European Childhood Obesity Project. $1 \mathrm{~m}(\mathrm{n}=64), 2 \mathrm{~m}(\mathrm{n}=91), 3 \mathrm{~m}(\mathrm{n}=91), 4 \mathrm{~m}(\mathrm{n}=97), 5 \mathrm{~m}(\mathrm{n}=96), 6 \mathrm{~m}(\mathrm{n}=96), 7 \mathrm{~m}(\mathrm{n}=96), 8 m(n=96), 9 m$ $(\mathrm{n}=93)$ and $12 \mathrm{~m}(\mathrm{n}=53)$.

were reported to contain cereals, representing 81 of the 225 evaluations. Comparisons are shown for all bottles assessed on the whole evaluation and for subanalyses on groups of bottles: bottles of formula correctly diluted with added cereals, bottles of formula diluted by not following the instructions (incorrectly diluted formula), and bottles of formula diluted by not following the instructions with powdered cereals added (incorrect preparation + cereals). Four columns show the results of the tests in percentages: (first) correctly by both methods, (second) incorrectly by both methods, (third) correctly by the SOPsystem and incorrectly without it, and (fourth) correctly without the SOPsystem and incorrectly with it.
The trained nutritionists had an $8.9 \%$ reduction in calculation errors in formula intake calculations and $18.5 \%$ in cereals (Table 2), whereas the nutrition students reduced by $43.8 \%$ errors in formula intake calculations and $55.6 \%$ in cereals (Table 3 ), both using the SOPsystem. In both cases, the absolute reduction of risk was the highest when the formula was diluted incorrectly and cereals were added.

The CV for each bottle was calculated per millilitre of formula and grams of cereal. Among trained nutritionists, the mean $\mathrm{CV}$ of the estimated formula was significantly lower $(P=0.005)$ using the SOPsystem $(\mathrm{CV}=0.007)$ compared with not using it $(\mathrm{CV}=0.014)$. The same pattern was observed for cereals

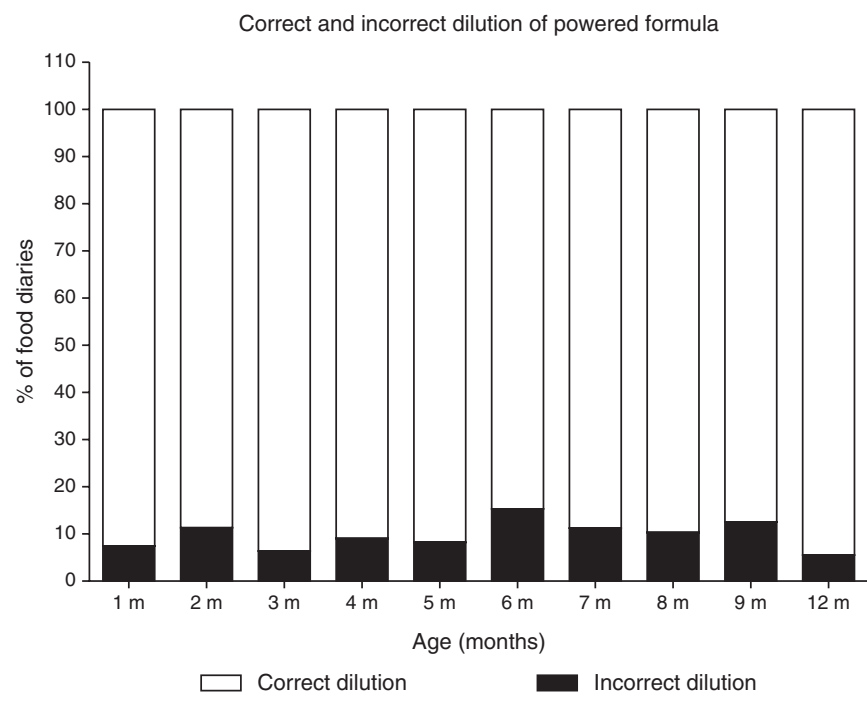

FIGURE 2. Frequency of correctly and incorrectly diluted formula milk in a random subsample of 10 food diaries of 100 infants in the European Childhood Obesity Project. $1 \mathrm{~m}(\mathrm{n}=68), 2 \mathrm{~m}(\mathrm{n}=97), 3 \mathrm{~m}(\mathrm{n}=96), 4 \mathrm{~m}(\mathrm{n}=100), 5 \mathrm{~m}(\mathrm{n}=98), 6 \mathrm{~m}(\mathrm{n}=98), 7 \mathrm{~m}(\mathrm{n}=98), 8 m(n=97), 9 m$ $(\mathrm{n}=96)$ and $12 \mathrm{~m}(\mathrm{n}=55)$. 
TABLE 2. Implementation of the SOPsystem among trained nutritionists: correct and incorrect calculations (\%) for each type of bottle using the SOPsystem (Excel Standardising Tool including SOPs equations) vs the no-SOPsystem (calculations performed using usual resources, such as a calculator)

\begin{tabular}{|c|c|c|c|c|c|c|c|c|}
\hline & Measure & $\begin{array}{c}\mathrm{n} \\
\text { Tests }\end{array}$ & $\begin{array}{l}\text { Correct } \\
\text { using both } \\
\text { methods } \\
\text { (n [\%]) }\end{array}$ & $\begin{array}{l}\text { Incorrect } \\
\text { using both } \\
\text { methods } \\
\text { (n [\%]) }\end{array}$ & $\begin{array}{c}\text { Correct } \\
\text { using manual } \\
\text { system only } \\
(\mathrm{n}[\%])\end{array}$ & $\begin{array}{c}\text { Correct } \\
\text { using SOP } \\
\text { system only } \\
\text { (n [\%]) }\end{array}$ & $\begin{array}{c}\text { Absolute } \\
\text { risk reduction } \\
(\%[95 \% \mathrm{CI}])\end{array}$ & $P^{\dagger}$ \\
\hline \multirow[t]{2}{*}{ Whole evaluation } & Formula & 225 & $193(85.8)$ & $4(1.8)$ & $4(1.8)$ & $24(10.7)$ & $8.9(3.9-13.8)$ & $<0.001$ \\
\hline & Cereals & 81 & $59(72.8)$ & $3(3.7)$ & $2(2.5)$ & $17(21.0)$ & $18.5(7.8-29.3)$ & 0.001 \\
\hline $\begin{array}{l}\text { Bottles correctly } \\
\text { diluted }+ \text { cereals }\end{array}$ & Formula & 45 & $36(80.0)$ & $2(4.4)$ & $0(0)$ & $7(15.6)$ & $15.6(2.4-28.7)$ & 0.016 \\
\hline $\begin{array}{l}\text { Bottles with incorrectly } \\
\text { diluted formula }\end{array}$ & Formula & 117 & $94(80.3)$ & $1(0.8)$ & $4(3.4)$ & $18(15.4)$ & $12.0(4.3-19.6)$ & 0.004 \\
\hline \multirow{2}{*}{$\begin{array}{l}\text { Bottles incorrectly } \\
\text { diluted }+ \text { cereals }\end{array}$} & Formula & 36 & $26(72.2)$ & $1(2.8)$ & $2(5.6)$ & $7(19.4)$ & $13.9(-2.4$ to 30.2$)$ & NS \\
\hline & Cereals & 34 & $25(69.4)$ & $0(0)$ & $0(0)$ & $9(25)$ & $26.5(11.2-41.7)$ & 0.004 \\
\hline
\end{tabular}

The defined correct answer was $\pm 2 \%$ the agreement value. $\mathrm{CI}=$ confidence interval; $\mathrm{NS}=$ not significant.

* Absolute risk reduction in proportion of bottles calculated incorrectly.

${ }^{\dagger} P$ value for McNemar test.

$(P<0.046, \mathrm{CV}=0.008$ for the SOPsystem and $\mathrm{CV}=0.015$ without the SOPsystem). The time required for the calculation was significantly shorter with the SOPsystem than without it (Table 4).

\section{DISCUSSION}

\section{Identification of Specific Sources of Error in Parental and Caregiver Preparation and Recording of Infants' Formula Milk Intake}

We detected a high frequency of parental errors in reporting total formula volumes and diluting the powdered formula. These results highlight the need to use the specific formatted FDs in studies of formula-fed infants, improving the description of bottle preparation and allowing the identification of misleading formula dilutions or misreporting of total volumes offered to the infants. Thus, an FD for formula-fed infants should collect data on both the amounts of water and powdered formula used to prepare the bottles, as well as data on total volumes offered and remaining after each meal.

These results also highlight the need to provide more education to parents in relation to the reconstitution of powdered formulas. Incorrect formula dilution may have important nutritional risks, including a possible increase in kidney workload (13) or an increased risk of obesity (14). Lucas et al (14) reported increased weights and fat mass estimates among infants fed powdered formula compared with infants fed with a ready-tofeed formula.

The high frequency of the reconstitution of formula deviating from manufacturers' instructions that we observed and the errors in total volume recorded by parents would lead to major differences in data interpretation unless a standardised approach to interpret FDs and determine the consumed amounts of reconstituted formula

TABLE 3. Implementation of the SOPsystem among inexperienced nutritionists: correct and incorrect calculations (\%) for each type using the SOPsystem (Excel Standardising Tool including SOPs equations) vs the no-SOPsystem (calculations performed using usual resources, such as a calculator)

\begin{tabular}{|c|c|c|c|c|c|c|c|c|}
\hline & Measure & $\begin{array}{c}\mathrm{n} \\
\text { Tests }\end{array}$ & $\begin{array}{l}\text { Correct } \\
\text { using both } \\
\text { methods } \\
\text { (n [\%]) }\end{array}$ & $\begin{array}{l}\text { Incorrect } \\
\text { using both } \\
\text { methods } \\
\text { (n [\%]) }\end{array}$ & $\begin{array}{c}\text { Correct } \\
\text { using manual } \\
\text { system only } \\
\text { (n [\%]) }\end{array}$ & $\begin{array}{c}\text { Correct } \\
\text { using SOP } \\
\text { system only } \\
\text { (n [\%]) }\end{array}$ & $\begin{array}{c}\text { Absolute risk } \\
\text { reduction } \\
(\%[95 \% \mathrm{CI}])\end{array}$ & $P^{\dagger}$ \\
\hline \multirow[t]{2}{*}{ Whole evaluation } & Formula & 575 & $161(28)$ & 74 (12.9) & $44(7.6)$ & $296(51.5)$ & $43.8(38.7-48.9)$ & $<0.001$ \\
\hline & Cereals & 207 & $45(21.7)$ & $31(15)$ & $8(3.9)$ & $123(59.4)$ & $55.6(47.6-63.5)$ & $<0.001$ \\
\hline $\begin{array}{l}\text { Bottles correctly } \\
\text { diluted + cereals }\end{array}$ & Formula & 115 & $38(33.3)$ & 13 (11.4) & $6(5.3)$ & $57(50)$ & $44.7(33.5-56.0)$ & $<0.001$ \\
\hline $\begin{array}{l}\text { Bottles with incorrectly } \\
\text { diluted formula }\end{array}$ & Formula & 299 & $19(6.3)$ & $52(17.4)$ & $2(0.7)$ & $226(75.6)$ & $74.9(69.7-80.1)$ & $<0.001$ \\
\hline \multirow{2}{*}{$\begin{array}{l}\text { Bottles incorrectly } \\
\text { diluted }+ \text { cereals }\end{array}$} & Formula & 92 & $0(0)$ & $16(17.4)$ & $0(0)$ & $76(82.6)$ & $82.6(74.7-90.5)$ & $<0.001$ \\
\hline & Cereals & 92 & $17(18.5)$ & $15(16.3)$ & $4(4.4)$ & $56(60.9)$ & $56.5(44.6-68.4)$ & $<0.001$ \\
\hline
\end{tabular}

The defined correct answer was $\pm 2 \%$ the agreement value. $\mathrm{CI}=$ confidence interval; NS $=$ not significant.

* Absolute risk reduction in proportion of bottles calculated incorrectly.

${ }^{\dagger} P$ value for McNemar test. 
TABLE 4. Comparison between mean time spent to interpret and calculate the dietary intake from the practice (simulated food diary) by both methods: with the SOPsystem and without it

\begin{tabular}{lcccr}
\hline & $\mathrm{n}$ & $\begin{array}{c}\text { Without SOPsystem, mean } \\
(\max -\min 95 \% \mathrm{CI}), \min \end{array}$ & $\begin{array}{c}\text { With SOPsystem, mean } \\
(\max -\min 95 \% \mathrm{CI})(\min )\end{array}$ & $\begin{array}{c}\text { Mean time difference } \\
(\max -\min 95 \% \mathrm{CI})\end{array}$ \\
\hline $\begin{array}{l}\text { Trained nutritionists and } \\
\quad \text { nonexpert nutrition students }\end{array}$ & 28 & $31.2(34.3-28.2)$ & $21.9(24.3-19.5)$ & $9.3(13.1-5.5)$ \\
$\begin{array}{l}\text { Nutritionists } \\
\text { Nonexpert nutrition students }\end{array}$ & 8 & $30.9(41.0-20.7)$ & $17.4(21.1-13.7)$ & $<0.001$ \\
\hline
\end{tabular}

$\mathrm{CI}=$ confidence interval.

* Paired samples $t$ test.

is applied. A possible weakness of this protocol may be the assumptions that should be performed in some situations. For instance, when the wrong dilution information was reported in FDs (the number of scoopfuls did not correspond to the water volume), but it was not possible to check with the study participants, it was assumed to be a dilution error, rather than an error in reporting water volume or the number of scoopfuls. This assumption could consider typing errors as dilution errors; however, this could also be considered strength of the protocol, ensuring consistent evaluation of FDs.

\section{Implementation of SOPs and the SOPsystem}

The different sequences of calculations required to determine dietary intake in different types of parental reports (if they do or do not take into account the increase in volume by the addition of powdered formula to the water, if they perform correct reconstitution of powdered formula, and combinations of both possibilities) enhance the need to standardise the evaluation of FDs. Moreover, the experience gained clearly shows the major advantages to using a software tool such as the SOPsystem to calculate dietary intakes of formula-fed infants in a multicentre setting, with respect to both the accuracy of the results and the work time needed to obtain these results.

The use of the SOPsystem for evaluation of infant formula intake consistently reduced observer error. Among nutritionists, the use of the SOPsystem was associated with a $>3$-fold success in performing the correct calculations. Among the less-experienced nutrition students, the use of the SOPsystem tool increased the probability of performing the calculations correctly, arriving at a 60 -fold higher probability of success when the formula was incorrectly diluted. The lower internutritionist CVs obtained when using the SOPsystem demonstrate that this tool guarantees the constant use of SOPs, unifies the methodology among the observers, and reduces the magnitude of error. These results suggest that the SOPsystem may improve the work of inexperienced nutritionists, reducing the time and effort needed for their training in FD evaluation in this age range. The use of the SOPsystem reduced the time needed for calculations to an average of $25.6 \%$ of the time needed for manual calculations in nutrition students and $44.1 \%$ in nutritionists. Although trained nutritionists needed the same time for manual calculations as nutrition students, they performed much faster calculations using the SOPsystem. The main limitation of the present study could be that all nutritionists performed the calculations without the SOPsystem first, and with the SOPsystem second. This could lead to a potential risk of training effect, partially magnifying the effect of the SOPsystem. This could partly explain how trainee nutritionists would benefit more than the experienced nutritionists would from the use of the SOPsystem.
Based on the results obtained in the present study, the use of the SOPsystem rather than manual calculations for evaluating the approximately nine thousand 3-day FDs obtained during the first year of life in the infants participating in the European Childhood Obesity Project, including reports on an estimated 121,500 feeding bottles, would result in an avoidance of 10,935 calculation errors and save approximately 1112 hours of work, which is $>7$ person-months.

It has not been assessed how the reduction in the error rate may influence total daily intake estimations. A $2 \%$ difference in calculations would guarantee a high precision; however, we cannot avoid the error produced by the lack of precision in the graduation of feeding bottles; this would have a major impact on total daily intake.

The easy implementation of the SOPsystem by expert and inexperienced nutritionists highlights its feasibility and easy adaptability to any study evaluating the intake of powdered formulas in infants. Furthermore, this procedure could also be used after data entry if all requested variables were entered in the database. This would increase the speed and efficacy of the nutritional evaluation of formula-fed infants and the interest of these established SOPs.

We conclude that the development and implementation of SOPs with a software tool that identified and corrected specific sources of error resulted in a harmonised and improved process for assessing dietary intake in formula-fed infants, minimising errors in the calculations and reducing the work time invested.

Acknowledgments: We gratefully acknowledge all the research nutritionists who have ever participated in the Childhood Obesity Project for all their hard work. We are extremely grateful to Joan Fernández-Ballart (from the Universitat Rovira i Virgili) and Pilar Hernández (IISPV) for their lessons and advice on statistical analyses.

\section{REFERENCES}

1. Livingstone MBE, Robson PJ, Wallace JMW. Issues in dietary intake assessment of children and adolescents. Br J Nutr 2004; 92:S213-22.

2. Potischman N, Cohen BE, Picciano MF. Dietary recommendations and identified research needs for the National Children's Study. J Nutr 2006;136:686-9.

3. Taren D, Dwyer J, Freedman L, et al. Dietary assessment methods: where do we go from here? Public Health Nutr 2002;5 (6A):1001-3.

4. Livingstone MBE, Robson PJ. Measurement of dietary intake in children. Proc Nutr Soc 2000;59:279-93.

5. Koletzko B, von Kries R, Closa R, et al. Lower protein in infant formula is associated with lower weight up to age $2 \mathrm{y}$ : a randomized clinical trial. Am J Clin Nutr 2009;89:1-10.

6. European Commission. Commission Directive 91/321/EEC of 14 May 1991 on infant and follow-on formulae. Off J Eur Union 1991:00350049 . 
7. Verwied-Jorky S, Schiess S, Luque V, et al. Methodology for longitudinal assessment of nutrient intake and dietary habits in early childhood in a transnational multicenter study. J Pediatr Gastroenterol Nutr 2011;52:96-102.

8. Burrows TL, Martin RJ, Collins CE. A systematic review of the validity of dietary assessment methods in children when compared with the method of doubly labeled water. J Am Diet Assoc 2010;110: $1501-10$.

9. Hill RJ, Davies PS. The validity of self-reported energy intake as determined using the doubly labelled water technique. Br J Nutr 2001;85:415-30.
10. Butte NF, Wong WW, Klein PD, et al. Measurement of milk intake: tracer-to-infant deuterium dilution method. Br J Nutr 1991;65:3-14.

11. Renfrew MJ, Ansell P, Macleod KL. Formula feed preparation: helping reduce the risks; a systematic review. Arch Dis Child 2003;88:855-8.

12. Schiess S, Grote V, Scaglioni S, et al. Introduction of complementary feeding in 5 European countries. J Pediatr Gastroenterol Nutr 2010;50:92-8.

13. Escribano J, Luque V, Ferre N, et al. Increased protein intake augments kidney volume and function in healthy infants. Kidney Int 2011;79:783-90.

14. Lucas A, Lockton S, Davies PS. Randomised trial of a ready-to-feed compared with powdered formula. Arch Dis Child 1992;67:935-9. 